\title{
SATB1 is an independent prognostic factor in radically resected upper gastrointestinal tract adenocarcinoma
}

\author{
Charlotta Hedner • Alexander Gaber • Dejan Korkocic • \\ Björn Nodin • Mathias Uhlén • Eugenia Kuteeva • \\ Henrik Johannesson • Karin Jirström • Jakob Eberhard
}

Received: 17 April 2014 /Revised: 12 August 2014 / Accepted: 6 October 2014 / Published online: 19 October 2014

(C) The Author(s) 2014. This article is published with open access at Springerlink.com

\begin{abstract}
Gastric cancer is the second most common cause of cancer-related death worldwide, and the incidence of esophageal adenocarcinoma is rising. While some progress has been made in treatment strategies, overall survival remains very poor for patients with adenocarcinoma in the upper gastrointestinal tract. Special AT-rich sequence binding protein 1 (SATB1) is a global genome organizer that has been demonstrated to promote aggressive tumor behavior in several different types of cancer, including gastric cancer. The prognostic value of SATB1 expression in esophageal cancer has, however, not yet been described. In this study, expression of SATB1 was examined by immunohistochemistry on tissue microarrays prepared from tissue samples from 175 patients with adenocarcinoma of the esophagus, cardia, or stomach and containing normal tissue, intestinal metaplasia, primary tumors, and metastases. A well-validated antibody was used. We found SATB1 to be an independent prognostic factor in patients with a radically resected tumor,
\end{abstract}

Electronic supplementary material The online version of this article (doi:10.1007/s00428-014-1667-6) contains supplementary material, which is available to authorized users.

C. Hedner $(\bowtie) \cdot$ A. Gaber $\cdot$ D. Korkocic $\cdot$ B. Nodin $\cdot$ K. Jirström • J. Eberhard

Department of Clinical Sciences, Division of Oncology and Pathology, Lund University, Skåne University Hospital, 221

85 Lund, Sweden

e-mail: charlotta.hedner@med.lu.se

M. Uhlén

Science for Life Laboratory, AlbaNova University Center, Royal Institute of Technology, 10691 Stockholm, Sweden

\section{Uhlén}

School of Biotechnology, AlbaNova University Center, Royal

Institute of Technology, 10691 Stockholm, Sweden

E. Kuteeva $\cdot$ H. Johannesson

Atlas Antibodies AB, AlbaNova University Center, 106

91 Stockholm, Sweden correlating with shorter overall survival as well as with shorter recurrence-free survival. SATB1 expression was also found to be significantly lower in primary tumors associated with intestinal metaplasia than those without intestinal metaplasia. This observation is of potential biological interest as it has been proposed that intestinal metaplasia-associated tumors constitute a less aggressive phenotype.

Keywords SATB1 · SATB2 - Gastric adenocarcinoma · Esophageal adenocarcinoma $\cdot$ Prognosis

\section{Introduction}

Gastric cancer was for a long time the leading cause of cancerrelated death worldwide [1]. Due principally to better living conditions, the incidence rate has been declining, but gastric cancer is still the second most common cause of cancer-related death worldwide [1-4] with 5-year survival rates ranging from 10 to $27 \%$ depending on the anatomical location and also on which part of the world the patient resides in [3]. In contrast, the incidence of esophageal adenocarcinomas has been steadily rising in the past decades $[2,5]$.

With such poor survival rates, there is an evident and immediate need to sharpen our diagnostic, prognostic, and treatment tools in order to improve survival rates for these patients. When it comes to treatment, there has been some progress during the last few years with large studies showing improved survival rates in patients receiving neoadjuvant or perioperative chemotheraphy and/or radiotherapy instead of surgical treatment only [6-8]. Although this is a step forward, there has been less progress in understanding the mechanisms that drive cancer progression and metastasis in these cancers and in the identification of clinically useful prognostic and treatment response predictive biomarkers. Hence, there is a need for novel biomarkers which might 
improve individualized treatment stratification and ultimately survival of patients with cancer in the upper gastrointestinal tract.

Special AT-rich sequence binding protein 1 (SATB1) is a global genome organizer [9] initially identified in thymocytes [10] and that recently attracted some attention as a putative cancer biomarker. Chromosomes are organized in the nucleus of a cell in such a way that only part of the genome is expressed [11]. This is regulated through chromatin proteins involved in chromatin compaction, which make chromatin fold into threedimensional structures which in turn determine which genes might be transcribed [9]. The genome harbors regions characterized by DNA sequences with one strand having A's, T's, and C's but no G's (ATC sequences, also known as BURs) [9]. When the ATC sequence context is disrupted by mutations, SATB1 binding is abolished [10]. SATB1 binds specifically to these ATC sequences, resulting in chromatin folding into loop domains which enables regulation of the expression of multiple genes $[9,11]$. Furthermore, SATB1 also provides a nuclear platform for docking of chromatin-remodeling enzymes, and through this mechanism, coordinates expression of several hundreds up to a thousand genes [9, 11, 12].

Expression of SATB1 has been correlated with a more aggressive tumor phenotype and worse prognosis in cancer of the breast [9, 12], ovary [13], colorectum [14-16], and larynx [17]. Han et al. suggested that its expression is necessary for breast cancer to become metastatic [12]. Other papers have reported contradicting results regarding the role of SATB1 in cancer progression in breast and colorectal cancer [18-20]. This may be due to differences in experimental design, e.g., examining SATB1 expression in total RNA transcripts from tumor tissue specimens as opposed to scoring SATB1 protein levels in individual tumor cells by immunohistochemistry [21]. In addition, differences in specificity of the antibodies used might significantly impact on the results.

SATB1 expression in gastric cancer has previously been examined in two studies on Chinese populations, both indicating that SATB1 expression is independently associated with worse prognosis $[22,23]$. An in vitro study supported the correlation between SATB1 expression and aggressive tumor behavior and also suggested that SATB1 plays a role in multidrug resistance [24]. SATB1 expression has, to our best knowledge, not been examined in esophageal cancer. The aim of our study was to examine longitudinal expression of SATB1 and its prognostic significance in adenocarcinomas of the esophagus, cardia, and stomach.

\section{Materials and methods}

Study design and participants

The study was performed on a consecutive cohort of 175 patients with adenocarcinoma in the upper gastrointestinal tract (esophagus, cardia, and stomach) who had been surgically treated in the university hospitals of Lund and Malmö from January 1, 2006-December 31, 2010. The cohort has been described in detail previously $[25,26]$. In brief, all tumors were histopathologically re-examined including confirmation of diagnosis, number of lymph nodes with metastasis (re-classified following the standardized TNM 7 classification by the American Joint Committee on Cancer (AJCC) [27]), and presence of intestinal metaplasia (Barrett's esophagus or gastric intestinal metaplasia (IM)) with or without dysplasia.

Clinical data, information on recurrence and vital status, or cause of death were obtained from the medical charts. The mean follow-up time for patients alive was 5.2 years (range 2.7-7.7).

Patient and tumor characteristics are provided in Supplemental Table 1.

Approval was obtained from the ethics committee at Lund University (ref no. 445-07).

\section{Tissue microarrays}

Tissue microarrays (TMAs) were constructed using a semiautomated arraying device (TMArrayer, Pathology Devices, Westminister, MD, USA) as previously described [25, 28]. Tissue was taken from viable, non-necrotic areas in duplicate 1-mm cores from primary tumors. In addition, lymph node metastases were sampled in 81 cases, IM (including Barrett's esophagus) in 73 cases, normal squamous epithelium in 96 cases, and normal gastric mucosa in 131 cases. Duplicate cores were obtained from different blocks of the primary tumor and different lymph node metastases in cases with more than one metastasis. Normal squamous epithelium and gastric mucosa were represented in single cores and IM in 1-3 cores.

\section{Antibody validation—Western blot}

Western blot analyses were performed according to standard protocols on SATB1 and SATB2 overexpression lysates coexpressed with a C-terminal myc-DDK tag $(\sim 3.1 \mathrm{kDa})$ in mammalian HEK293T cells (LY427355 and LY414656, respectively, Origene Technologies, Rockville, MD, USA). Briefly, $2 \mu 1$ of SATB1 and SATB2 overexpression lysate was separated on precast 4-20\% CriterionTGX SDS-PAGE gradient gels (Bio-Rad Laboratories, Hercules, CA) under reducing conditions, followed by blotting to PVDF membranes (Trans-Blot ${ }^{\circledR}$ Turbo ${ }^{\mathrm{TM}}$ Midi PVDF Transfer Packs, Bio-Rad Laboratories, Hercules, CA), according to the instructions of the manufacturer. Membranes were blocked for 45 min at RT in blocking buffer ( $5 \%$ dry milk, $0.5 \%$ Tween $20,1 \times$ TBS) prior to addition of antibody (anti-SATB1, clone EPR3895, Epitomics, Burlingame, CA, USA; anti-SATB2 \#AMAb90679 CL0320, Atlas Antibodies AB, Stockholm, 
Sweden; or anti-DDK Tag\# TA50011, Origene Technologies, Rockville, MD, USA), diluted to a final concentration of $1 \mu \mathrm{g} / \mathrm{ml}$ in blocking buffer. Following incubation for $1 \mathrm{~h}$ with primary antibody, the membranes were washed $4 \times 5 \min$ in $1 \times$ TBS with $0.1 \%$ Tween 20 . Horseradish peroxidase (HRP)conjugated secondary antibody (swine anti-rabbit antibody \#P0399 or goat anti-mouse antibody \#P0447, Dako), diluted 1:3,000 in blocking buffer, was added to the membranes and incubated for 30 min followed by a final round of washing. Detection was carried out using chemiluminescence HRP substrate (Immobilon, EMD Millipore Corporation, Billerica, MA, USA) according to the manufacturer's instructions.

\section{Antibody validation —immunohistochemistry}

The specificity of SATB1 and SATB2 antibodies was further evaluated in immunohistochemical experiments.

Tissue sections $(4 \mu \mathrm{m})$ were cut from TMAs containing 18 normal (fallopian tube, cervix, endometrium, placenta, testis, prostate, liver, pancreas, rectum, colon, stomach, duodenum, small intestine, cerebellum, cerebral cortex, skin, skeletal muscle, and tonsil) and 7 cancer (prostate, colorectal, ventricular, renal, liver, lung, and breast) tissues. Prior to immunostaining, the sections were baked at $50{ }^{\circ} \mathrm{C}$ overnight and deparaffinized in xylene and graded ethanol. Antigen retrieval was then performed using citrate buffer $\mathrm{pH} 6$ (ThermoFisher Scientific, Waltham, MA, USA) in decloaking chamber (Biocare Medical, Walnut Creek, CA, USA). Sections were stained with anti-SATB1 rabbit monoclonal antibody (Clone EPR3895, Epitomics, Burlingame, CA, USA) diluted 1:100 or mouse monoclonal antibody against SATB2 (AMAb90679, CL0320, Atlas Antibodies, Stockholm, Sweden) diluted 1:1,000 in Autostainer 480S (ThermoFisher Scientific, Waltham, MA, USA) using a commercial kit (UltraVision LP HRP polymer ${ }^{\circledR}$, Primary Antibody Enhancer, Ultra V Block and DAB plus substrate system ${ }^{\circledR}$, ThermoFisher Scientific, Waltham, MA, USA). Slides were counterstained with hematoxylin and mounted using Pertex.

Slides were examined, and images were taken using an automated system (VSlide, Metasystems).

Immunohistochemistry and staining evaluation

For immunohistochemistry, 4- $\mu \mathrm{m}$ TMA sections were baked in a heated chamber for $120 \mathrm{~min}$ at $60^{\circ} \mathrm{C}$. Antigen retrieval for Ki67, p53, and SATB1 was performed using HIER pH 9 (PTlink system Dako, Glostrup, Denmark), and for SATB2 pH 6 (decloaking chamber, Biocare Medical, Walnut Creek, CA, USA).

For Ki67, a monoclonal antibody (clone MIB1 Dako, diluted 1:50) was applied in a BenchMark ULTRA (Ventana Medical systems, Tuscon, AZ, USA).
Expression of p53 was analyzed using a monoclonal antibody (clone DO-7, Dako). Expression of SATB1 was assessed using a monoclonal antibody (Clone EPR 3895, Epitomics, Burlingame, CA, USA, diluted 1:100), as for SATB2 (AMAb90679 CLO320, Atlas Antibodies, diluted 1:1,000), and staining for all three antibodies was performed in an Autostainer Plus (Dako, Glostrup, Denmark). DAB was used as chromogen, and the slides were counterstained with hematoxylin.

For assessment of Ki67 expression, the fraction of Ki67 nuclear staining was categorized as follows: $0-1,2-10,11-$ $20,21-50$, and $>50 \%$. For statistical analysis, three categories were applied: $0-20,21-50$, and $>50 \%$.

The fraction of p 53 staining was categorized as follows: 0 $1,2-10,11-50$, and $>50 \%$. For statistical analysis, three categories were applied: $0-1,2-50$, and $>50 \%$.

The estimated fraction of cells with nuclear SATB1 expression was denoted and after that, transformed into five categories of $0(0-1 \%), 1(2-25 \%), 2(26-50 \%), 3(51-75 \%)$, and $4(>75 \%)$. The predominant nuclear intensity was estimated as negative (0), weak (1), moderate (2), or strong (3). For statistical analysis, a combined nuclear score was constructed by multiplying fraction and intensity, and any intensity of staining of $\geq 2 \%$ of the cells was denoted as positive SATB1 staining. In line with previous studies, stromal lymphocytes served as a positive control for SATB1 [9]. Evaluation of nuclear SATB2 expression was recorded in the same manner as described for SATB1.

All stained sections were evaluated by two independent observers who were blinded to clinical and outcome data.

\section{Statistical analysis}

The chi-squared test was applied to analyze the relationship between SATB1 expression and clinicopathological parameters. Overall survival (OS) rates and recurrence-free survival (RFS) time according to SATB1 negativity versus SATB1 positivity were calculated using Kaplan-Meier analysis. To assess differences in the Kaplan-Meier curves, the log-rank test was used. Unadjusted and adjusted hazard ratios (HR) for OS and RFS were calculated by Cox regression proportional hazard modeling. The adjusted model included age, sex, $\mathrm{T}$ stage, $\mathrm{N}$ stage, $\mathrm{M}$ stage, differentiation, and SATB1 expression.

For some subjects, information on one or several markers was not available. Missing values were coded as a separate category for categorical variables. Missing values for categorical variables co-varied. The adjusted model did not converge due to many constant values. In order to avoid this, only patients with information on SATB1 expression were included in the adjusted analysis. 
A backward conditional method was used for variable selection in the adjusted model.

For all analyses, IBM SPSS Statistics version 20.0 (SPSS Inc., Chicago, IL, USA) was used. $p$ values $<0.05$ were considered significant. All tests were two-sided.

\section{Results}

\section{Antibody validation}

Western blot analyses were performed on HEK293T cell lysates overexpressing the full-length SATB1 and SATB2 proteins (Fig. 1a-c) and revealed that both antibodies bind specifically and selectively to their respective target protein.

Different staining patterns for SATB1 and SATB2 were obtained on normal and cancer tissues. SATB1 immunoreactivity was limited to a subpopulation of lymphoid cells in various tissues (Fig. 1d, f), but no immunoreactivity was observed in glandular cells in the rectum (Fig. 1d), colon, or in colorectal cancer (data not shown). In addition, weak to moderate nuclear staining was seen in single cells in the fallopian tube, seminiferous tubules, and in the majority of glandular cells in prostate. Strong nuclear immunoreactivity was detected in single neurons in cerebral cortex.

Very strong immunoreactivity for SATB2 was observed in colorectal mucosa (Fig. 1e) as well as in colorectal cancer (data not shown). Moderate nuclear positivity was seen in a subset of neurons in cerebral cortex and single glandular cells in the duodenum, kidney, and prostate. In tonsil, only single lymphoid cells displayed very weak nuclear immunoreactivity (Fig. 1g).

Taken together, IHC and Western blot validation demonstrates that the two antibodies used in this study are highly specific to their respective target protein, despite extensive sequence similarity of the two proteins.

\section{Longitudinal SATB1 expression}

SATB1 could be evaluated in 71/96 (74\%) samples with normal squamous epithelium, 125/131 (95\%) samples with normal gastric mucosa, 63/73 (86\%) samples with IM, 170/175 (97\%) primary tumors, and 79/81 (98\%) metastases. Immunohistochemical images are shown in Fig. 2.

As demonstrated in Fig. 3a, SATB1 expression was significantly higher in primary tumors $(n=53 / 170,31.2 \%)$ and metastases $(n=32 / 79,40.5 \%)$ than in normal squamous epithelium $(n=0 / 96,0 \%)$ and normal gastric mucosa $(n=0 / 131$, $0 \%$ where no expression was seen. SATB1 expression did not differ between the primary tumors and metastases $(p=$ $0.116)$. SATB1 expression was significantly higher in IM than that in normal tissue $(p=0.003)$, but the number of SATB1expressing IM samples was very small $(n=8)$. Figure $3 \mathrm{~b}$ shows that the expression of SATB1 was significantly lower in primary tumors with tumor-associated IM than that in primary tumors without tumor-associated IM ( $p=$ 0.031 ), but this difference was not maintained in metastases.

Correlations of SATB1 expression in primary tumors with clinicopathological parameters

Table 1 lists the distribution of clinicopathological and investigative parameters according to SATB1 expression. Significant associations of SATB1 expression were found with younger age $(p=0.045)$ and more advanced $\mathrm{N}$ stage $(p=0.010)$. SATB1 expression was also significantly more common in esophageal compared to cardiac or gastric cancer $(p=0.033)$.

\section{Impact of SATB1 expression on survival}

Kaplan-Meier analysis of radically resected (R0) tumors revealed both decreased OS $(p=0.033)$ and shorter RFS $(p=$ 0.021 ) for patients with SATB1-positive compared to SATB1negative tumors (Fig. 4). SATB1expression was significantly associated with shorter RFS in patients with a R0 tumor and distant metastasis-free (M0) disease $(p=0.008)$ but not with OS.

As demonstrated in Table 2, the prognostic value of SATB1 was confirmed in unadjusted Cox regression analysis for OS in patients with $\mathrm{R} 0$ resection $(\mathrm{HR}=1.74 ; 95 \% \mathrm{CI} 1.04-2.90$, $p=0.036)$ and for RFS in patients with R0 resection/M0 disease $(\mathrm{HR}=2.53 ; 95 \%$ CI 1.24-5.16, $p=0.011)$. These associations remained significant in adjusted analysis $(\mathrm{HR}=$ 2.30; $95 \%$ CI 1.32-4.01, $p=0.003$ for OS and $\mathrm{HR}=3.88$; $95 \%$ CI $1.72-8.72, p=0.001$ for RFS), and also when tumor location was included in the adjusted model (data not shown).

In the entire cohort, SATB1 positivity was not significantly associated with overall survival but with a significantly shorter RFS in unadjusted (HR $=1.68 ; 95 \%$ CI 1.04-2.71, $p=0.032)$ but not in adjusted analysis (data not shown).

As shown in Supplemental Fig. 1, SATB2 was expressed to a very limited extent in the examined tissues. No correlation with RFS or OS was seen (data not shown), and hence, no further statistical analyses were performed. Expression of Ki67 and p53 in primary tumors and metastases had no prognostic significance (data not shown). Kaplan-Meier analysis revealed a trend toward longer OS for patients with tumor-adjacent IM compared with patients without tumoradjacent IM ( $p=0.054$, data not shown). Patients with R0 tumors had a prolonged OS compared with patients with non-R0 tumors ( $p=<0.001$, data not shown). 
Fig. 1 Assessment of specificity of anti-SATB1 and anti-SATB2 antibodies using Western blot (ac) and immunohistochemistry (de) analyses. Western blot results following incubation with antiSATB1 (a), anti-SATB2 (b), or anti-DDK Tag (c) antibodies (lane 1 , molecular weight marker; lane 2 , negative control lysate; lane 3 , SATB1; and lane 4, SATB2overexpressing mammalian HEK293T lysate). Note specific binding of antibodies to their respective lysates.

Immunohistochemistry results following staining with antiSATB1 (d, f) and anti-SATB2 (e, g) antibodies in rectum $(\mathbf{d}, \mathbf{e})$ and tonsil (f, g). Note strong nuclear immunoreactivity in a subset of lymphocytes following staining with anti-SATB1 antibody both in rectum and tonsil and absence of nuclear immunoreactivity in glandular epithelium of rectum. Staining with anti-SATB2 displays strong nuclear positivity in rectum glandular cells, while lymphoid cells are mainly negative
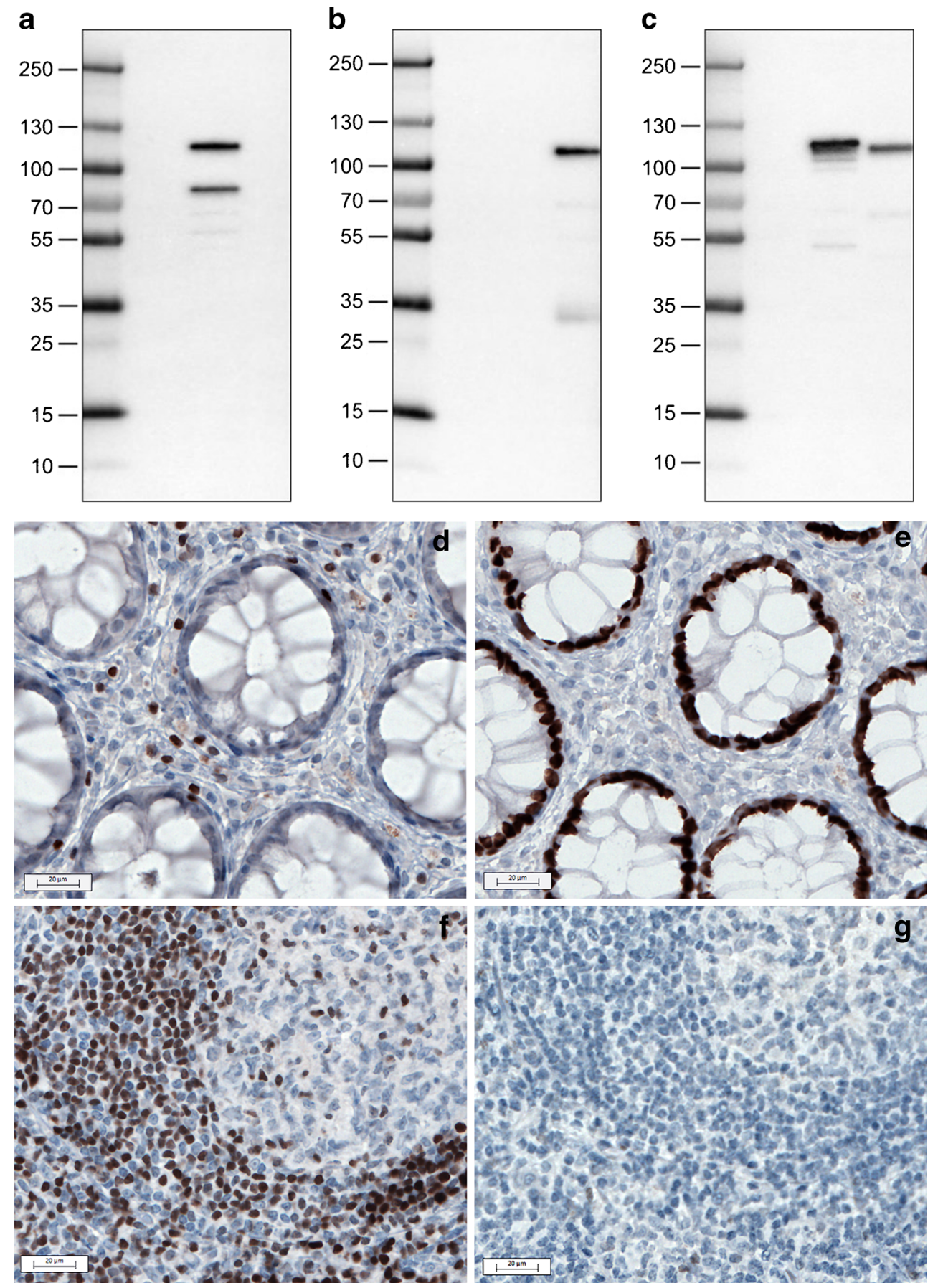

\section{Discussion}

We have examined the expression of SATB1 in matched normal squamous epithelium, normal gastric mucosa, Barrett's esophagus, gastric intestinal metaplasia, and primary and metastatic adenocarcinoma in patients with cancer of the upper gastrointestinal tract with known clinical outcome. We show that SATB1 expression in primary tumors is an independent prognostic marker for shorter OS and shorter RFS in patients with radically resected tumors. These results are in line with several previous studies indicating that SATB1 expression correlates with a more aggressive phenotype and worse prognosis in several types of cancer [9, 12-16].

In our cohort, involvement of resection margins was significantly associated with poor prognosis. This not only validates the use of the cohort for biomarker studies but also strengthens the prognostic value of SATB1 expression in radically resected tumors, in particular since an earlier study was limited by a lack of information on residual tumor after surgery [13]. SATB1 expression was prognostic for both OS 
a
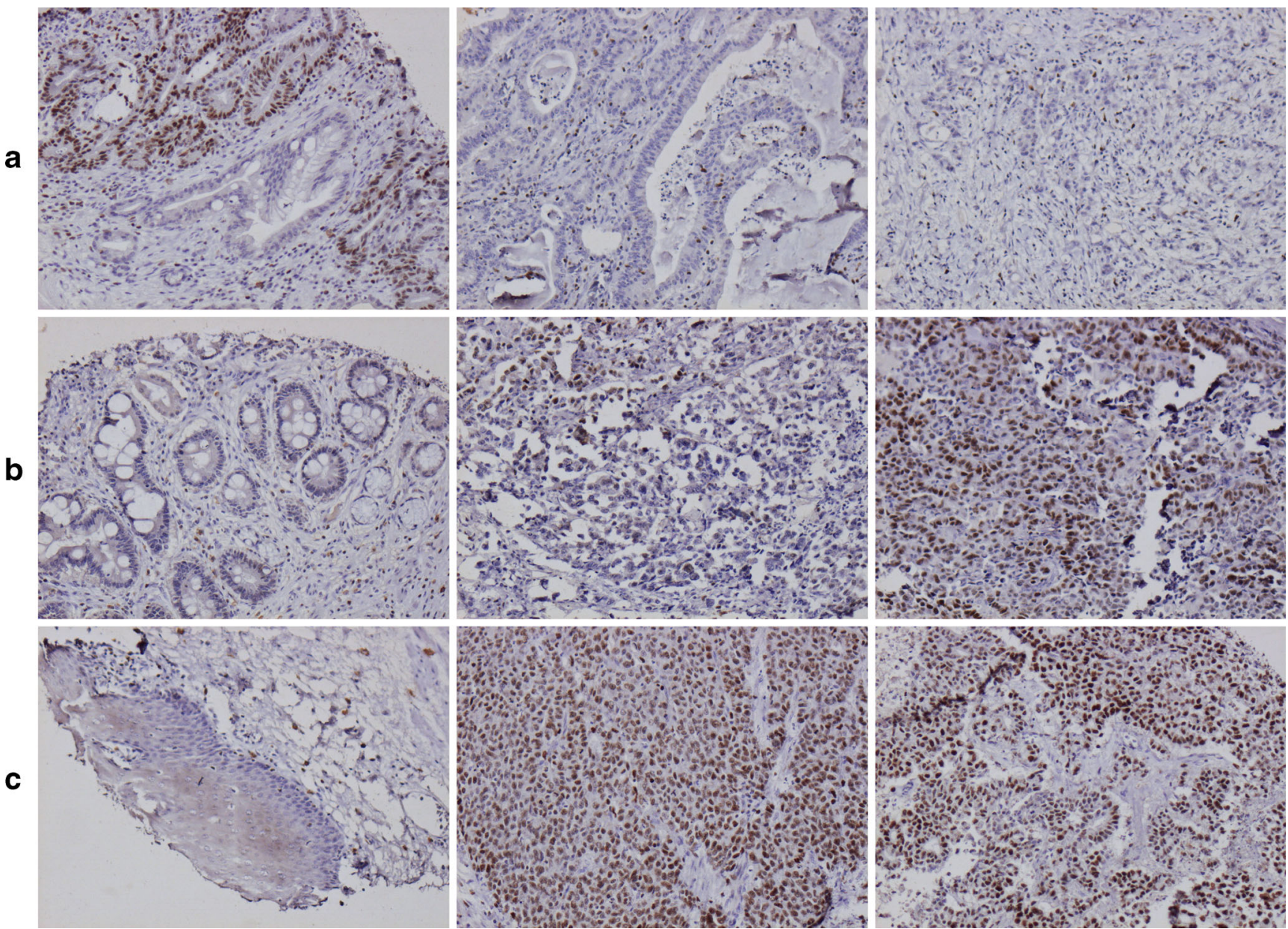

Fig. 2 Examples of immunohistochemical SATB1 staining. Images $(\times 10$ magnification) of SATB1 expression in different tissue entities from three cases. From left to right (a) low- and high-grade dysplastic intestinal metaplasia (score 4), cancer (score 0 ), and metastasis (score 0 but with
SATB1-positive lymphocytes) in a T2N2M0 esophageal cancer; (b) intestinal metaplasia (score 1), cancer (score 4), and metastasis (score 9) in a T3N1M0 cardiac cancer; (c) normal squamous epithelium (score 0), cancer (score 12), and metastasis (score 12) in a T3N3M0 cardiac cancer a

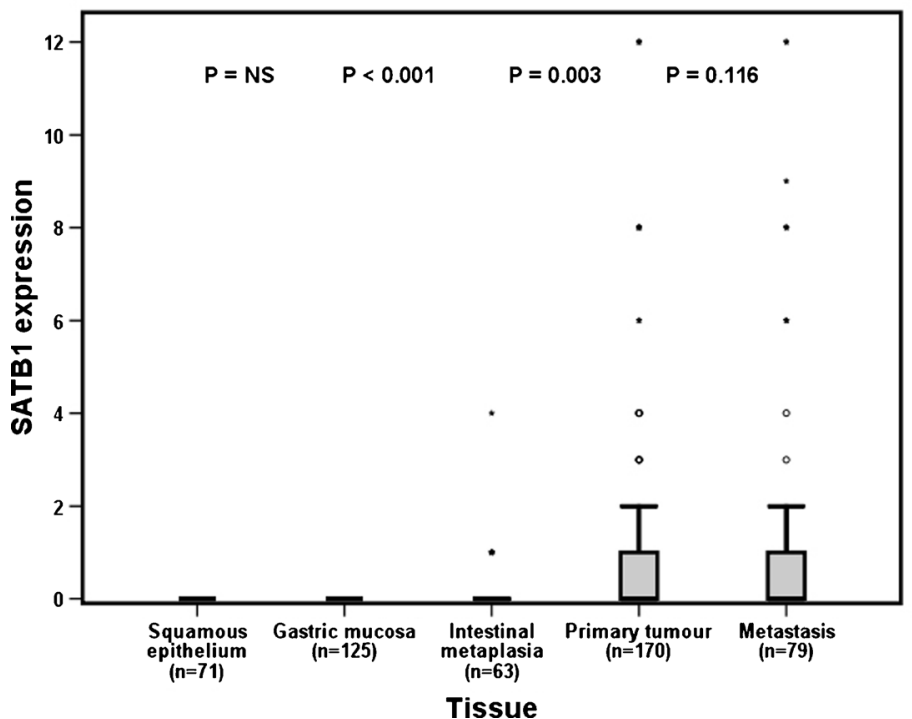

b

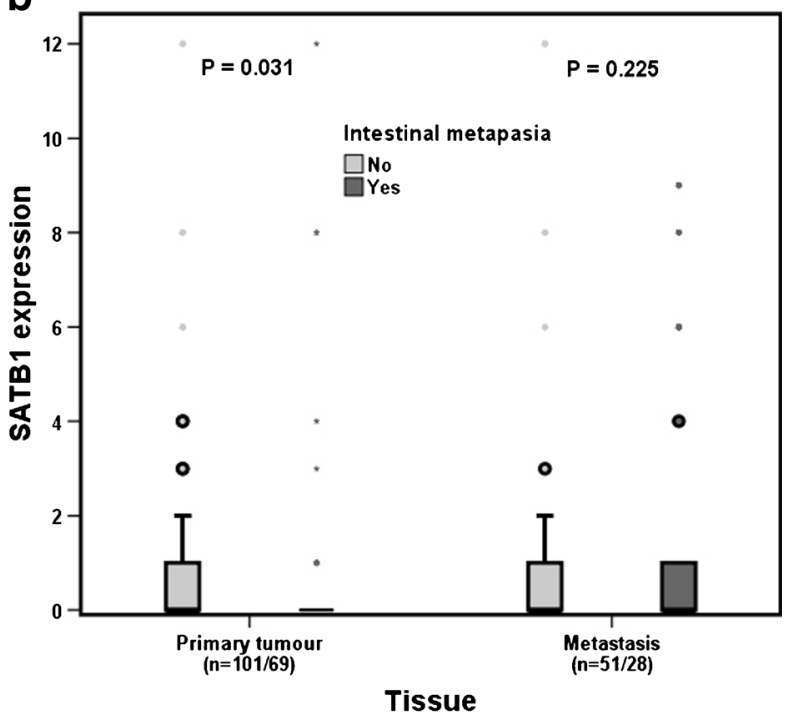

Fig. 3 Visualization of SATB1 expression according to tissue type. a SATB1 expression according to tissue type in the entire cohort. b SATB1 expression in primary tumors (left) and metastases (right) with and without the presence of intestinal metaplasia (Barrett's esophagus included) 
Table 1 Distribution of clinicopathological characteristics according to SATB1 expression

\begin{tabular}{|c|c|c|c|c|c|c|c|}
\hline \multicolumn{3}{|l|}{ SAIBI } & & \multirow{4}{*}{ Factor, $n(\%)$} & \multirow{2}{*}{\multicolumn{2}{|c|}{ SATB1 expression }} & \multirow{4}{*}{$p$ value } \\
\hline \multirow{4}{*}{ Factor, $n(\%)$} & \multirow{2}{*}{\multicolumn{2}{|c|}{ SATB1 expression }} & \multirow{4}{*}{$p$ value } & & & & \\
\hline & & & & & Negative, 117 & Positive, 53 & \\
\hline & \multirow{2}{*}{$\begin{array}{l}\text { Negative, } 117 \\
(68.9)\end{array}$} & \multirow{2}{*}{$\begin{array}{l}\text { Positive, } 53 \\
(31.2)\end{array}$} & & & $(68.9)$ & $(31.2)$ & \\
\hline & & & & $2-50 \%$ & $30(256)$ & $11(208)$ & \\
\hline \multicolumn{3}{|l|}{ Age } & \multirow[t]{3}{*}{0.045} & $>50 \%$ & $50(42.7)$ & $31(58.5)$ & \\
\hline Mean & 71.4 & 67.9 & & Missing & 1 & 0 & \\
\hline Median (range) & $72.9(42.6-94.4)$ & $65.6(48.2-87.2)$ & & SATB2 expression & & & $<0.001$ \\
\hline \multicolumn{3}{|l|}{ Sex } & \multirow[t]{3}{*}{0.492} & SATB2-negative & $109(93.2)$ & $38(71.7)$ & \\
\hline Women & $30(25.6)$ & $11(20.8)$ & & SATB2-positive & $8(6.8)$ & $15(28.3)$ & \\
\hline Men & $87(74.4)$ & $42(79.2)$ & & Resection margin & & & 0.271 \\
\hline \multicolumn{3}{|l|}{ T stage } & \multirow[t]{4}{*}{0.243} & R0 & $82(70.1)$ & $37(69.8)$ & \\
\hline 1 & $11(9.6)$ & $7(13.2)$ & & R1 & $27(23.1)$ & $6(11.3)$ & \\
\hline 2 & $28(24.6)$ & $4(7.5)$ & & $\mathrm{R} 2$ & $8(6.8)$ & $10(18.9)$ & \\
\hline 3 & $58(50.9)$ & $32(60.4)$ & & & & & \\
\hline
\end{tabular}

and RFS in R0 tumors only when all R0 tumors were included. When R0 tumors with distant metastasis (M1) were excluded, SATB1 was only significantly associated with OS, but this might change with a longer follow-up time. SATB1 expression was not different between primary tumors and metastases, indicating that it is sufficient to examine only the primary tumor for prognostic purposes.

We evaluated SATB1 expression using an approach similar to that used in a previous study [22]. As in previous studies, we found that lymphocytes are suitable as internal positive control $[9,18]$. We also confirm that expression of SATB1 in a limited fraction of the tumor cells already confers poor prognosis [12].

The prognostic value of SATB1 is controversial in different tumor types, which may be due to tissue-dependent regulatory functions of SATB1 [18]. Possible implications of differences in methodologies and materials also need to be considered, notably the possibility of discordance between messenger RNA (mRNA) and protein levels [29]. Immunohistochemistry (IHC) allows assessment of protein expression of a putative biomarker in specific cell types and even its subcellular location. Our results are in line with those reported by Han et al., in that expression of SATB1 protein independently predicts worse outcome [12]. Studies on SATB1 mRNA levels failed to demonstrate independent prognostic value for SATB1 expression [19, 20]. Such mRNA studies usually include both tumor and normal cells, which is an inherent source of error. For biomarker studies therefore, IHC is a more reliable method of investigation. The use of different antibodies is another potential source of controversy. SATB1 is highly homologous to SATB2 and the specificity of SATB1 and SATB2 antibodies has been questioned in previous studies [18]. The specificity of antibodies for SATB1 and SATB2 needs to be thoroughly validated [9], as we have performed in this study. This is all the more important as 

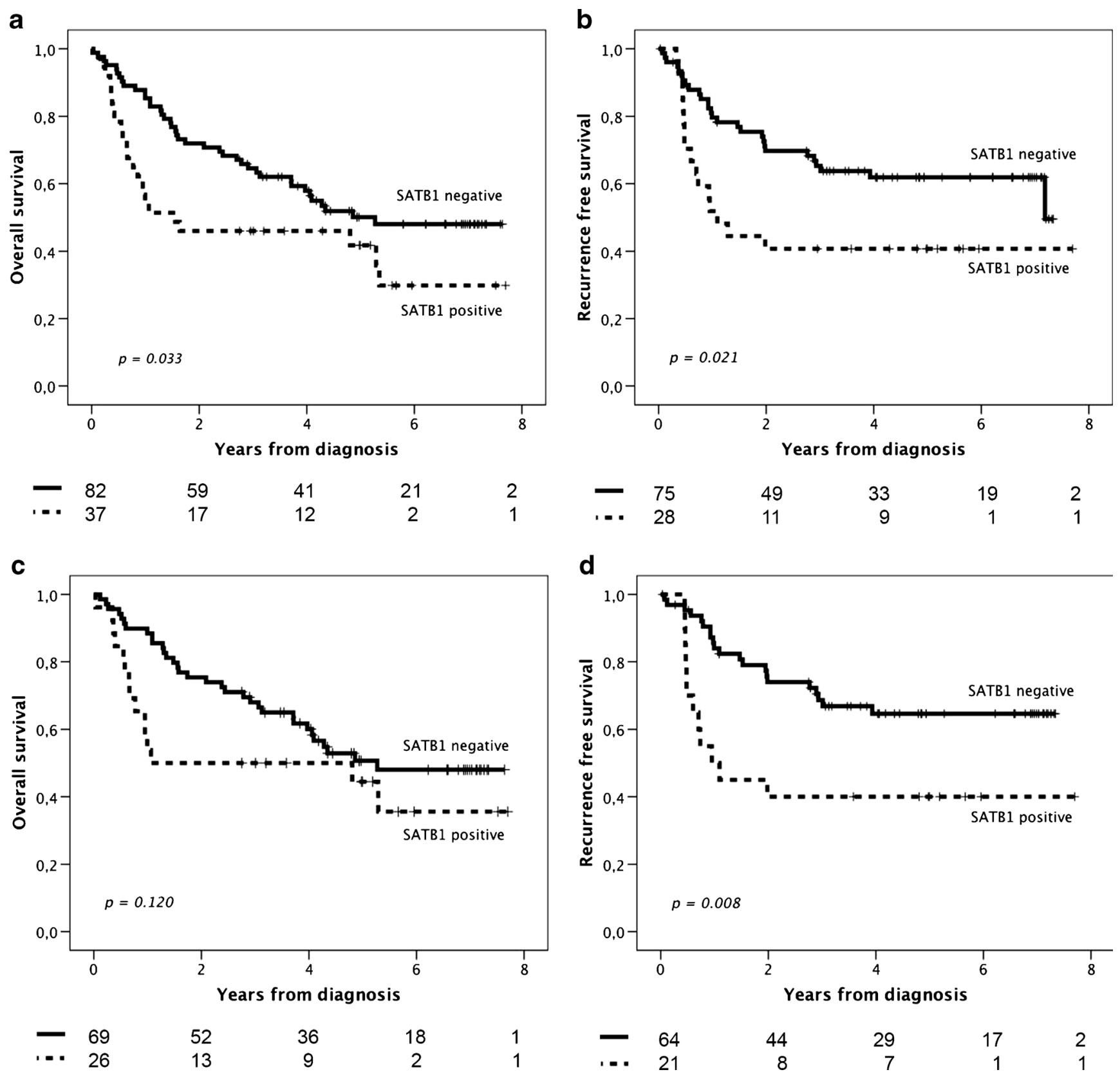

Fig. 4 Kaplan-Meier estimates of survival and recurrence according to SATB1 expression. Overall survival (a) and recurrence-free survival (b), according to SATB1 expression in patients with radically resected tumors.

several studies have indicated that SATB1 and SATB2 have antagonistic qualities $[9,14,30]$. As an example, SATB2 expression was a favorable prognostic marker in colorectal cancer [30, 31].

SATB1 remained significant as a prognostic factor when tumor location was included in the adjusted Cox regression model, indicating that SATB1 is prognostic in upper gastrointestinal tract cancer regardless of its anatomical location. Interestingly, SATB1 expression was significantly lower in primary tumors associated with IM than in primary tumors not associated with IM. This is in line with at least two different

Overall survival (c) and recurrence-free survival (d) in patients with radically resected tumors and distant metastasis-free disease

pathways of gastroesophageal carcinogenesis, one intestinal (arising from dysplasia in IM) and one non-intestinal (arising from cardia-type mucosa), the former being associated with better overall survival [32]. Our cohort showed a similar trend, which is in agreement with SATB1 expression as a negative prognostic factor.

ERBB2 (HER2) is an important drug target in breast cancer and an increasingly important target in gastric cancer [8]. SATB1 upregulates ERBB2 (HER2) expression [9, 12], which draws attention to SATB1 as a candidate drug target. Knockdown of SATB1 in aggressive breast cancer cell lines 


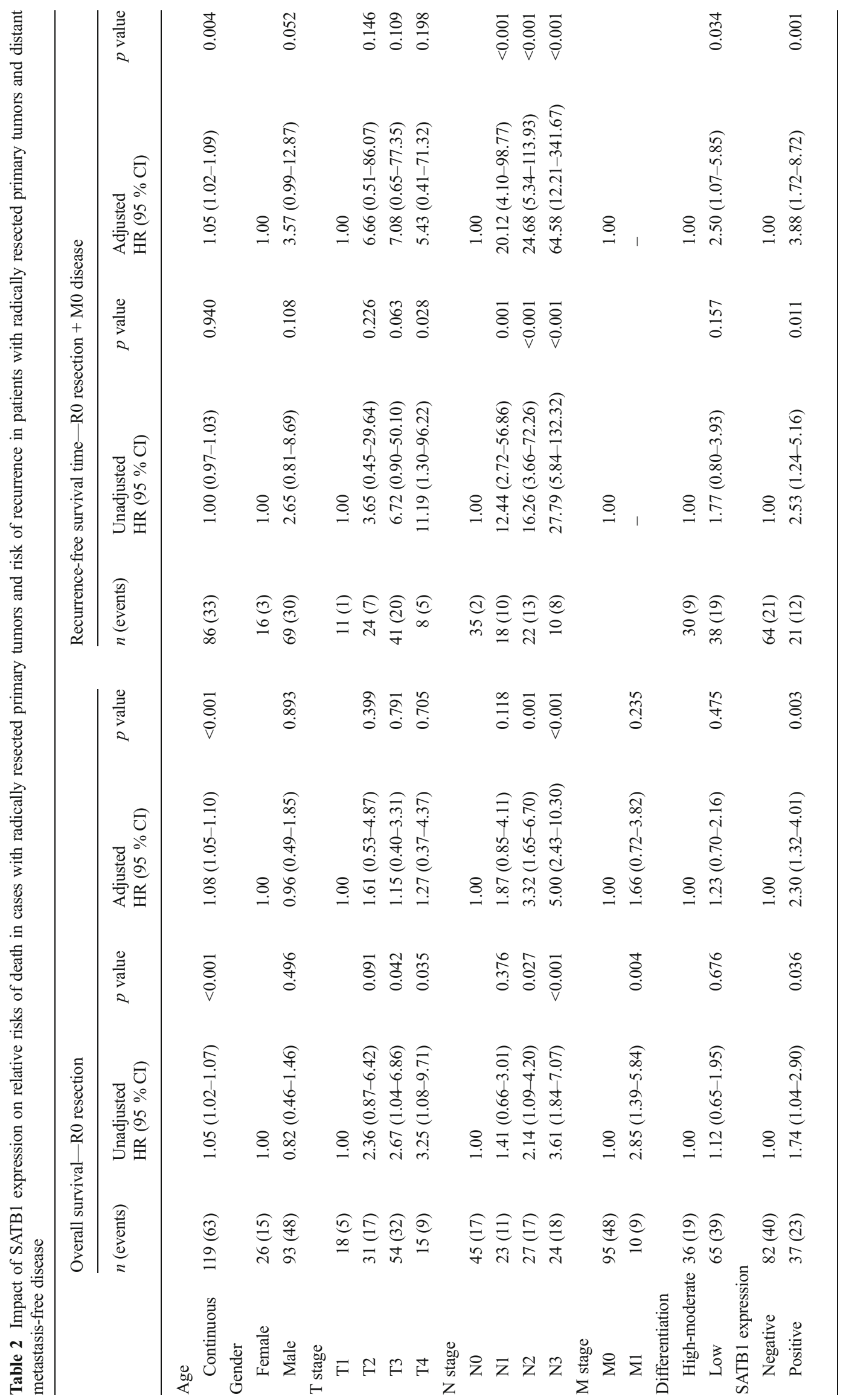


caused complete reversal of tumor growth and metastatic abilities in vivo and introduction of SATB1 decoy DNA drastically reduced invasive and metastatic capacity of SATB1-positive cell lines [33]. Similar results were reported in colorectal cancer $[9,12,16]$. SATB1 downregulates expression of E-cadherin, which is a characteristic event in epithelial to mesenchymal transition and an important step in invasion and metastasis $[34,35]$.

Studies, using the same well-validated anti-SATB1 antibody as in our study, have indicated that SATB1 contributes to chemotherapy multidrug resistance [24], which provides additional arguments in favor of SATB1-blocking as a novel therapeutic approach.

None of the patients in this study had received neoadjuvant treatment. This rules out any possibility that biomarker expression was affected by treatment, which must be considered a strength of this study. A further strength is that all available surgically treated tumors were included consecutively, which excludes risk of selection bias. A limitation of the present study is the use of TMAs with a risk of sampling bias. Our TMA design limits this as duplicate cores were taken from different blocks of the primary tumor and different lymph node metastases in cases with more than one metastasis. Furthermore, even with full-face sections, sampling bias is not excluded as these also represent only a limited fraction of the tumor. An advantage of the TMA approach is the high number of tumors that can be studied, which conceivably might compensate for false negative or positive tissue cores [36].

In conclusion, we show that SATB1 is an independent prognostic biomarker in patients with radically resected adenocarcinomas of the upper gastrointestinal tract.

\begin{abstract}
Acknowledgments This study was supported by grants from the Knut and Alice Wallenberg Foundation, the Swedish Cancer Society, the Crafoord Foundation, the Olle Engkvist Foundation, Anna Lisa and Sven-Eric Lundgren's Foundation, Lund University Faculty of Medicine, and University Hospital Research Grants.
\end{abstract}

Conflict of interest The authors declare that they have no competing interests.

Open Access This article is distributed under the terms of the Creative Commons Attribution License which permits any use, distribution, and reproduction in any medium, provided the original author(s) and the source are credited.

\section{References}

1. Bertuccio P, Chatenoud L, Levi F, Praud D, Ferlay J, Negri E, Malvezzi M, La Vecchia C (2009) Recent patterns in gastric cancer: a global overview. Int J Cancer 125(3):666-673

2. Lauwers GY (2009) Epithelial neoplasms of the stomach. In:Odze RD, Goldblum JR (eds) Surgical pathology of the GI tract, liver, biliary tract and pancreas, volume 1, 2nd edn. Elsevier, Philadelphia, p 563-580

3. Parkin DM, Bray F, Ferlay J, Pisani P (2005) Global cancer statistics, 2002. CA Cancer J Clin 55(2):74-108

4. Ferlay J, Shin HR, Bray F, Forman D, Mathers C, Parkin DM (2010) Estimates of worldwide burden of cancer in 2008: GLOBOCAN 2008. Int J Cancer 127(12):2893-2917

5. Devesa SS, Blot WJ, Fraumeni JF Jr (1998) Changing patterns in the incidence of esophageal and gastric carcinoma in the United States. Cancer 83(10):2049-2053

6. Cunningham D, Allum WH, Stenning SP, Thompson JN, Van de Velde CJ, Nicolson M, Scarffe JH, Lofts FJ, Falk SJ, Iveson TJ et al (2006) Perioperative chemotherapy versus surgery alone for resectable gastroesophageal cancer. N Engl J Med 355(1):11-20

7. van Hagen $P$, Hulshof MC, van Lanschot JJ, Steyerberg EW, van Berge Henegouwen MI, Wijnhoven BP, Richel DJ, Nieuwenhuijzen GA, Hospers GA, Bonenkamp JJ et al (2012) Preoperative chemoradiotherapy for esophageal or junctional cancer. N Engl J Med 366(22):2074-2084

8. Bang YJ, Van Cutsem E, Feyereislova A, Chung HC, Shen L, Sawaki A, Lordick F, Ohtsu A, Omuro Y, Satoh T et al (2010) Trastuzumab in combination with chemotherapy versus chemotherapy alone for treatment of HER2-positive advanced gastric or gastro-oesophageal junction cancer (ToGA): a phase 3, open-label, randomised controlled trial. Lancet 376(9742):687-697

9. Kohwi-Shigematsu T, Poterlowicz K, Ordinario E, Han HJ, Botchkarev VA, Kohwi Y (2013) Genome organizing function of SATB1 in tumor progression. Semin Cancer Biol 23(2):72-79

10. Dickinson LA, Joh T, Kohwi Y, Kohwi-Shigematsu T (1992) A tissue-specific MAR/SAR DNA-binding protein with unusual binding site recognition. Cell 70(4):631-645

11. Yasui D, Miyano M, Cai S, Varga-Weisz P, Kohwi-Shigematsu T (2002) SATB1 targets chromatin remodelling to regulate genes over long distances. Nature 419(6907):641-645

12. Han HJ, Russo J, Kohwi Y, Kohwi-Shigematsu T (2008) SATB1 reprogrammes gene expression to promote breast tumour growth and metastasis. Nature 452(7184):187-193

13. Nodin B, Hedner C, Uhlen M, Jirstrom K (2012) Expression of the global regulator SATB1 is an independent factor of poor prognosis in high grade epithelial ovarian cancer. J Ovarian Res 5(1):24

14. Nodin B, Johannesson H, Wangefjord S, O'Connor DP, Lindquist KE, Uhlen M, Jirstrom K, Eberhard J (2012) Molecular correlates and prognostic significance of SATB1 expression in colorectal cancer. Diagn Pathol 7:115

15. Meng WJ, Yan H, Zhou B, Zhang W, Kong XH, Wang R, Zhan L, Li Y, Zhou ZG, Sun XF (2012) Correlation of SATB1 overexpression with the progression of human rectal cancer. Int J Color Dis 27(2): $143-150$

16. Zhang J, Zhang B, Zhang X, Sun Y, Wei X, McNutt MA, Lu S, Liu Y, Zhang D, Wang M et al (2013) SATB1 expression is associated with biologic behavior in colorectal carcinoma in vitro and in vivo. PLoS ONE 8(1):e47902

17. Zhao XD, Ji WY, Zhang W, He LX, Yang J, Liang HJ, Wang LL (2010) Overexpression of SATB1 in laryngeal squamous cell carcinoma. ORL J Otorhinolaryngol Relat Spec 72(1):1-5

18. Al-Sohaily S, Henderson C, Selinger C, Pangon L, Segelov E, Kohonen-Corish M, Warusavitarne J (2013) Loss of special AT-rich sequence-binding protein 1 (SATB1) predicts poor survival in patients with colorectal cancer. Histopathology 65(2):155-63. doi: 10.1111/his. 12295

19. Iorns E, Hnatyszyn HJ, Seo P, Clarke J, Ward T, Lippman M (2010) The role of SATB1 in breast cancer pathogenesis. J Natl Cancer Inst 102(16):1284-1296

20. Hanker LC, Karn T, Mavrova-Risteska L, Ruckhaberle E, Gaetje R, Holtrich U, Kaufmann M, Rody A, Wiegratz I (2011) SATB1 gene expression and breast cancer prognosis. Breast 20(4):309-313 
21. Kohwi-Shigematsu T, Han HJ, Russo J, Kohwi Y (2010) Re: The role of SATB1 in breast cancer pathogenesis. J Natl Cancer Inst 102(24):1879-1880, author reply 1880-1871

22. Lu X, Cheng C, Zhu S, Yang Y, Zheng L, Wang G, Shu X, Wu K, Liu K, Tong Q (2010) SATB1 is an independent prognostic marker for gastric cancer in a Chinese population. Oncol Rep 24(4):981-987

23. Cheng C, Lu X, Wang G, Zheng L, Shu X, Zhu S, Liu K, Wu K, Tong $\mathrm{Q}$ (2010) Expression of SATB1 and heparanase in gastric cancer and its relationship to clinicopathologic features. APMIS 118(11):855863

24. Sun F, Lu X, Li H, Peng Z, Wu K, Wang G, Tong Q (2012) Special AT-rich sequence binding protein 1 regulates the multidrug resistance and invasion of human gastric cancer cells. Oncol Lett 4(1):156-162

25. Fristedt R, Gaber A, Hedner C, Nodin B, Uhlen M, Eberhard J, Jirstrom K (2014) Expression and prognostic significance of the polymeric immunoglobulin receptor in esophageal and gastric adenocarcinoma. J Transl Med 12(1):83

26. Jonsson L, Hedner C, Gaber A, Korkocic D, Nodin B, Uhlen M, Eberhard J, Jirstrom K (2014) High expression of RNA-binding motif protein 3 in esophageal and gastric adenocarcinoma correlates with intestinal metaplasia-associated tumours and independently predicts a reduced risk of recurrence and death. Biomark Res 2:11

27. Edge SB, Compton CC (2010) The American Joint Committee on Cancer: the 7th edition of the AJCC cancer staging manual and the future of TNM. Ann Surg Oncol 17(6):1471-1474

28. Kononen J, Bubendorf L, Kallioniemi A, Barlund M, Schraml P, Leighton S, Torhorst J, Mihatsch MJ, Sauter G, Kallioniemi OP (1998) Tissue microarrays for high-throughput molecular profiling of tumor specimens. Nat Med 4(7):844-847
29. Larsson A, Fridberg M, Gaber A, Nodin B, Leveen P, Jonsson G, Uhlen M, Birgisson H, Jirstrom K (2012) Validation of podocalyxinlike protein as a biomarker of poor prognosis in colorectal cancer. BMC Cancer 12:282

30. Wang S, Zhou J, Wang XY, Hao JM, Chen JZ, Zhang XM, Jin H, Liu L, Zhang YF, Liu J et al (2009) Down-regulated expression of SATB2 is associated with metastasis and poor prognosis in colorectal cancer. J Pathol 219(1):114-122

31. Eberhard J, Gaber A, Wangefjord S, Nodin B, Uhlen M, Ericson Lindquist K, Jirstrom K (2012) A cohort study of the prognostic and treatment predictive value of SATB2 expression in colorectal cancer. Br J Cancer 106(5):931-938

32. Demicco EG, Farris AB 3rd, Baba Y, Agbor-Etang B, Bergethon K, Mandal R, Daives D, Fukuoka J, Shimizu M, Dias-Santagata D et al (2011) The dichotomy in carcinogenesis of the distal esophagus and esophagogastric junction: intestinal-type vs cardiac-type mucosa-associated adenocarcinoma. Mod Pathol 24(9):1177-1190

33. Yamayoshi A, Yasuhara M, Galande S, Kobori A, Murakami A (2011) Decoy-DNA against special AT-rich sequence binding protein 1 inhibits the growth and invasive ability of human breast cancer. Oligonucleotides 21(2):115-121

34. Gheldof A, Berx G (2013) Cadherins and epithelial-to-mesenchymal transition. Prog Mol Biol Transl Sci 116:317-336

35. Fang XF, Hou ZB, Dai XZ, Chen C, Ge J, Shen H, Li XF, Yu LK, Yuan Y (2013) Special AT-rich sequence-binding protein 1 promotes cell growth and metastasis in colorectal cancer. World J Gastroenterol 19(15):2331-2339

36. Torhorst J, Bucher C, Kononen J, Haas P, Zuber M, Kochli OR, Mross F, Dieterich H, Moch H, Mihatsch M et al (2001) Tissue microarrays for rapid linking of molecular changes to clinical endpoints. Am J Pathol 159(6):2249-2256 